\title{
The emperor has some clothes
}

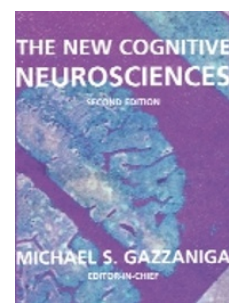

The New Cognitive Neurosciences

by M. S. Gazzaniga

MIT Press, Cambridge, Massachusetts, 1999. \$129.95

hardcover, pp 1276

ISBN 0-262-07195-9

\section{Reviewed by Jochen Braun}

The New Cognitive Neurosciences was a vast undertaking, the hefty fruit of about 180 contributors' labors. Just like its predecessor, the book surveys the field of cognitive neuroscience, which attempts to explain all aspects of mental life in terms of brain processes. The book does not have any close rivals, and its roster of distinguished contributors almost guarantees its continued prominence in the field. The New Cognitive Neuroscience likely provides the most representative cross-section of cognitive neuroscience that is possible at this time. Thus, it is an ideal starting point for graduate students or anyone else wishing to get oriented in the field.

The material of The New Cognitive Neuroscience is organized into 11 sections, each of which is meant to be a mini-textbook for one area. Two sections are particularly successful in this respect: Development (section editor, P. Rakic) and Language (W. J. M. Levelt). Their chapters are well organized, and their authors review each topic without unnecessarily emphasizing their own work. My favorites in the Development section were the 'basic principles' overview (Ch. 1, Rakic) and the chapter on the superior colliculus (Ch. 5, Stein, Wallace \& Stanford), which presents asides on cognitive issues, such as the possible relevance of multi-sensory integration to synesthesia. In the Language section, I was impressed by the chapter on 'core word production' (Ch. 59, Indefrey \& Levelt) and by the comparison of different types of computational models in language research (Ch. 64, Saffran, Dell \& Schwartz).

The auditory chapters of Sensory Systems (Movshon \& Blakemore) are strong, particularly the sections on the avian song system (Ch. 32, Doupe, Brainard \& Hessler; Ch. 33, Doya \&

Jochen Braun is Professor of Cognitive and Theoretical Neurobiology at the University of Plymouth, Drake Circus, Plymouth, Devon PL4 $8 A A, U K$.

e-mail:achim@klab.caltech.edu
Sejnowski), and the encoding of timbre in primary auditory cortex (Ch. 29, Shamma). Motor Systems (Bizzi) is also excellent, especially its sections on motor planning or intentions (Ch. 36, Andersen et al.; Ch. 38, Rizzolatti, Fogassi \& Gallese), learning of sequential movements (Ch. 39, Hikosaka et al.), and the role of hippocampal place cells in rodent naviation (Ch. 41, Wilson). Evolution (Cosmides \& Tooby) might have served as the logical point of departure for the entire book, as its section editors so persuasively argue. In this section, I found the chapters on 'theory of mind' tests for autistic children (Ch. 85, Leslie), and on content-free and socially based reasoning (Ch. 87, Tooby \& Cosmides) particularly interesting. Consciousness (Schacter) astonishes by rising to the difficulty of the topic. Its highlights include the historical overview by the section editor, the balanced assessment of various empirical measures of conscious perception (Ch. 90, Merikle \& Danemann) and the comparison of sleep and waking states (Ch. 93, Hobson, Pace-Schott \& Stickgold). The visual sections of Sensory Systems, Attention (DiGirolamo \& Posner), Memory (Tulving) and Higher Cognitive Functions (Kosslyn \& Smith) are not as strong. Here, section editors were less successful in organizing and subdividing their topics, and most chapters simply focus on authors' research. Nonetheless, individual chapters are often excellent.

Only two sections truly disappoint. Plasticity (Black) evades almost every first-order question the reader brings to the topic. What are the various manifestations of plasticity and their characteristic time courses? What are possible subcellular substrates? How is plasticity restricted to appropriate sites and a plasticity catastrophe avoided? Some chapters border on the perfunctory. (Surely, Aplysia holds more lessons than are told here.) Much the same can be said for the section Emotion (LeDoux). Here, the reader is catapulted into minutiae such as how the amygdala responds to the ingestion of salted oranges, and how post-traumatic stress damages the hippocampus. Surely, emotions have something to do with feelings? Here we learn nothing about phenomenal aspects of emotion. And isn't the adaptive value of emotion to be sought in the communicative/social sphere? Nothing here reveals how emotions are shared or socially triggered.

This book provides two larger messages. The first is that the shining successes of cognitive neuroscience tend to concern evolutionary adaptations. Consider the perception of faces and facial signals in the inferotemporal cortex (Ch. 28), the planning and mental rehearsal of movement in the premotor and posterior parietal cortex, the integration of different sensory modalities in the superior colliculus (Ch. 5, Ch. 31), the sense of place and direction in the hippocampus and thalamus, and the acquisition, rehearsal and production of bird song. In each instance, the study of an ancestrally relevant skill has been rewarded by the discovery of specific anatomical substrates. As the editors of the evolution section rightly insist, brains are organized according to an evolutionary logic and can be understood only once this logic is firmly grasped.

The second message is that the field of cognitive neuroscience is now an emperor with at least some clothes. It has been said, with fair justification, that "The dirty secret of contemporary neuroscience ... is [that] we do not have a unifying theoretical principle ... the central objective of the now majestic research program in neuroscience remains beyond reach: there is only the most shaky understanding of how the brain ... engenders mind-the capacity to reflect on past events, to think, and to imagine" (John Searle, The Mystery of Consciousness. New York Review of Books, Nov. 2 \& 16, 1995). Although there is no central theory, cognitive neuroscience now offers local theories of how the brain causes adaptive skills such as planning a movement, integrating different senses, or learning to sing like one's father. These local theories create solid links between neuronal, algorithmic and behavioral levels and go well beyond a "shaky understanding." For this reason, the book's emphasis on local theories is especially satisfying, and its sober avoidance of the loftier types of computational theorizing is wise. Thus, the true message of The New Cognitive Neuroscience is the persuasive explanatory power of local theories of specific adaptive skills. 